\title{
COMMENTS ON THE KKM THEORY ON HYPERCONVEX METRIC SPACES
}

\author{
SEHIE PARK
}

\begin{abstract}
Recent results in the KKM theory on abstract convex spaces and the related multimap classes $\mathfrak{K C}$ and $\mathfrak{K O}$ are applied to deduce generalizations of results on KKM maps in metric spaces in Amini et al. [1] and generalized KKM theorems on hyperconvex metric spaces in Chang et al. $[4,5]$.
\end{abstract}

\section{Introduction}

The notion of hyperconvex metric spaces was introduced by Aronszajn and Panitchpakdi [2] in 1956. Later, in 1979, independently Sine [31] and Soardi [34] proved that a bounded hyperconvex metric space has the fixed point property for nonexpansive maps. Since then many interesting works related to nonexpansive maps have appeared for hyperconvex metric spaces; see [13].

In 1996, Khamsi [11] established the Knaster-Kuratowski-Mazurkiewicz theorem (in short, KKM theorem) [15] for hyperconvex metric spaces and applied it to obtain a Schauder type fixed point theorem. This line of study has been followed by a number of authors; see [13, 18, 19, 20] and references therein. Especially, the present author obtained extensions or equivalent forms of the KKM theorem, a Fan-Browder type fixed point theorem, and other results for hyperconvex metric spaces in $[18,19,20]$. Moreover, Kirk et al. [14] established the KKM theorem, its equivalent formulations, fixed point theorems, and their applications for hyperconvex metric spaces.

Later, it is known that most of the results in the KKM theory of hyperconvex metric spaces are simple consequences of much more general results. In fact, Horvath [8, 9] initiated study of the KKM theory and fixed point theory for $c$-spaces or $H$-spaces, which are significant generalizations of convex spaces or convex subsets of topological vector spaces. Moreover, in [9], he found that hyperconvex metric spaces are particular $c$-spaces. On the other hand, the present author initiated study of the KKM theory on generalized convex spaces or $G$-convex spaces, which properly include the class of $c$-spaces and a large number of spaces having particular type of abstract convexity; see $[17,21,22,27,32]$ and references therein.

Received March 11, 2009.

2000 Mathematics Subject Classification. 47H04, 47H10, 49J27, 49J35, 54C60, 54H25, 91B50.

Key words and phrases. Abstract convex space, KKM space, generalized $(G-)$ convex space, hyperconvex metric space. 
SEHIE PARK

Recently, we generalized $G$-convex spaces to abstract convex spaces and introduced the multimap classes $\mathfrak{K C}$ and $\mathfrak{K O}$ related to generalizations of KKM maps; see [23, 24, 25, 26, 28, 29, 30, 31]. As applications of our new KKM theory on abstract convex spaces, in this paper, we deduce generalizations of recent results on KKM maps for metric spaces in Amini et al. [1] and generalized KKM theorems on hyperconvex metric spaces in Chang et al. $[3,4,5,6]$.

In Section 2, we introduce the concepts of abstract convex spaces, KKM spaces, and multimap classes $\mathfrak{K C}$ and $\mathfrak{K O}$. Section 3 deals with four basic theorems in the KKM theory on abstract convex spaces. In Section 4, main results in [1] are extended to $\mathfrak{K C}$ maps and $\mathfrak{K O}$-maps. Section 5 deals with KKM maps in hyperconvex metric space. In fact, we show that any hyperconvex metric space is a KKM space and that all results in $[4,5]$ can be generalized.

\section{Abstract convex spaces and the map classes $\mathfrak{K C}$ and $\mathfrak{K O}$}

Let $\langle D\rangle$ denote the set of all nonempty finite subsets of a set $D$. Multimaps are also called simply maps.

Definition. $[23,24,25,26,28,29,30,31]$ An abstract convex space $(E, D ; \Gamma)$ consists of a topological space $E$, a nonempty set $D$, and a multimap $\Gamma:\langle D\rangle \multimap E$ with nonempty values. We may denote $\Gamma_{A}:=\Gamma(A)$ for $A \in\langle D\rangle$.

For any $D^{\prime} \subset D$, the $\Gamma$-convex hull of $D^{\prime}$ is denoted and defined by

$$
\operatorname{co}_{\Gamma} D^{\prime}:=\bigcup\left\{\Gamma_{A} \mid A \in\left\langle D^{\prime}\right\rangle\right\} \subset E
$$

A subset $X$ of $E$ is called a $\Gamma$-convex subset of $(E, D ; \Gamma)$ relative to $D^{\prime}$ if for any $N \in\left\langle D^{\prime}\right\rangle$, we have $\Gamma_{N} \subset X$, that is, $\operatorname{co}_{\Gamma} D^{\prime} \subset X$. Then $\left(X, D^{\prime} ;\left.\Gamma\right|_{\left\langle D^{\prime}\right\rangle}\right)$ is called a $\Gamma$-convex subspace of $(E, D ; \Gamma)$.

When $D \subset E$, the space is denoted by $(E \supset D ; \Gamma)$. In such case, a subset $X$ of $E$ is said to be $\Gamma$-convex if $\operatorname{co}_{\Gamma}(X \cap D) \subset X$; in other words, $X$ is $\Gamma$-convex relative to $D^{\prime}:=X \cap D$. In case $E=D$, let $(E ; \Gamma):=(E, E ; \Gamma)$.

\section{Example.}

1. A generalized convex space or a $G$-convex space $(X, D ; \Gamma)$ due to Park consists of a topological space $X$, a nonempty set $D$, and a multimap $\Gamma:\langle D\rangle \multimap X$ such that for each $A \in\langle D\rangle$ with the cardinality $|A|=n+1$, there exists a continuous function $\phi_{A}: \Delta_{n} \rightarrow \Gamma(A)$ such that $J \in\langle A\rangle$ implies $\phi_{A}\left(\Delta_{J}\right) \subset \Gamma(J)$.

Here, $\Delta_{n}$ is the standard $n$-simplex with vertices $\left\{e_{i}\right\}_{i=0}^{n}$, and $\Delta_{J}$ the face of $\Delta_{n}$ corresponding to $J \in\langle A\rangle$; that is, if $A=\left\{a_{0}, a_{1}, \ldots, a_{n}\right\}$ and $J=\left\{a_{i_{0}}, a_{i_{1}}, \ldots, a_{i_{k}}\right\} \subset$ $A$, then $\Delta_{J}=\operatorname{co}\left\{e_{i_{0}}, e_{i_{1}}, \ldots, e_{i_{k}}\right\}$. For details, see [17, 21, 22, 27, 32] and references therein. 
2. A $G$-convex space $(X, D ; \Gamma)$ is called an $H$-space if each $\Gamma_{A}$ is $\omega$-connected (that is, $n$-connected for all $n \geq 0)$ and $\Gamma_{A} \subset \Gamma_{B}$ for $A \subset B$ in $\langle D\rangle$. An $H$-space reduces to a $c$-space due to Horvath whenever $X=D$. The concepts of $c$-spaces, l.c.-spaces, and l.c.-metric spaces were extensively studied by Horvath $[8,9]$.

3. A convex space $(X, D ; \Gamma)$ is a triple where $X$ is a subset of a vector space, $D \subset X$ such that $\operatorname{co} D \subset X$, and each $\Gamma_{A}$ is the convex hull of $A \in\langle D\rangle$ equipped with the Euclidean topology. This concept generalizes the one due to Lassonde [16] for $X=D$.

4. For further examples of abstract convex spaces, see [31].

Definition. Let $(E, D ; \Gamma)$ be an abstract convex space and $Z$ a topological space. For a multimap $F: E \multimap Z$ with nonempty values, if a multimap $G: D \multimap Z$ satisfies

$$
F\left(\Gamma_{A}\right) \subset G(A):=\bigcup_{y \in A} G(y) \quad \text { for all } A \in\langle D\rangle,
$$

then $G$ is called a KKM map with respect to $F$. A KKM map $G: D \multimap E$ is a KKM map with respect to the identity map $1_{E}$.

A multimap $F: E \multimap Z$ is called a $\mathfrak{K C}$-map [resp., a $\mathfrak{K O}-$ map] $[23,24,25,26]$ if, for any closed-valued [resp., open-valued] KKM map $G: D \multimap Z$ with respect to $F$, the family $\{G(y)\}_{y \in D}$ has the finite intersection property. We denote

$$
\mathfrak{K C}(E, Z):=\{F: X \multimap Z \mid F \text { is a } \mathfrak{K C}-\text { map }\} .
$$

Similarly, $\mathfrak{K O}(E, Z)$ is defined. Some authors use the notation $\operatorname{KKM}(E, Z)$ instead of $\mathfrak{K C}(E, Z)$.

\section{Remark.}

1. If $1_{E} \in \mathfrak{K C}(E, E)$, then $f \in \mathfrak{K C}(E, Z)$ for any continuous function $f: E \rightarrow Z$. This also holds for $\mathfrak{K O}$.

2. For any topological space $Y$, if $F: E \rightarrow Y$ is a continuous single-valued map or if $F: F \multimap Y$ has a continuous selection, then $F \in \mathfrak{K C}(E, Y) \cap \mathfrak{K O}(E, Y)$.

3. For more details on the classes $\mathfrak{K C}$ and $\mathfrak{K O}$, see [23, 24, 25, 26, 30, 31].

Definition. An abstract convex space $(E, D ; \Gamma)$ is said to satisfy the $K K M$ principle if, for any closed-valued [resp., open-valued] KKM map $G: D \multimap E$, the family $\{G(y)\}_{y \in D}$ has the finite intersection property; that is, the identity map $1_{E} \in \mathfrak{K C}(E, E) \cap \mathfrak{K O}(E, E)$.

A KKM space is an abstract convex space satisfying the KKM principle.

Example. We give examples of KKM spaces:

1. Every $G$-convex space is a KKM space; see [21, 29, 30, 31]. 
2. A connected linearly ordered space $(X, \leq)$ can be made into an abstract convex topological space $(X \supset D ; \Gamma)$ for any nonempty $D \subset X$ by defining $\Gamma_{A}:=[\min A, \max A]$ $=\{x \in X \mid \min A \leq x \leq \max A\}$ for each $A \in\langle D\rangle$. Further, it is a KKM space; see $[26$, Theorem $5(\mathrm{i})]$.

3. The extended long line $L^{*}$ can be made into a KKM space $\left(L^{*} \supset D ; \Gamma\right)$; see [26]. But $L^{*}$ is not a $G$-convex space.

4. For a triple $(H, X ; \Gamma)$, where $H$ is a hyperconvex metric space, $X \subset H$, and $\Gamma_{A}:=$ $\bigcap\{B \mid B$ is a closed ball containing $A\}$ for $A \in\langle X\rangle$, Khamsi [11] showed $1_{H} \in$ $\mathfrak{K C}(H, H)$. Horvath [9] showed that a hyperconvex metric space is an l.c. space, a particular type of $H$-space. Therefore, it is a KKM space.

5. Let $X$ be a closed convex subset of a complete $\mathbb{R}$-tree $H$. Then $H$ is a hyperconvex metric space. Let $G: X \multimap H$ be a map with nonempty closed [resp., open] values such that, for each finite $A \in\langle X\rangle$,

$$
\operatorname{conv}_{H}(A) \subset G(A),
$$

where $\operatorname{conv}_{H}(A)$ is the intersection of all closed convex subsets of $H$ that contain $A$. Then $\{G(x)\}_{x \in X}$ has the finite intersection property; see Kirk and Panyanak [12] and Park [31]. Hence, $\left(H, X ; \operatorname{conv}_{H}\right)$ is a KKM space.

Theorem 0. [30] Let $(X, D ; \Gamma)$ be a KKM space and $G: D \multimap X$ a closed-valued $K K M$ map. If

(1) $\bigcap_{z \in M} G(z)$ is compact for some $M \in\langle D\rangle$,

then we have

$$
\bigcap_{z \in D} G(z) \neq \emptyset .
$$

\section{Basic theorems in the KKM theory}

In our KKM theory on abstract convex spaces given in [23, 29], there exist some basic theorems from which we can deduce several equivalent formulations that can be used for applications. In this section, we introduce some of such basic theorems.

We begin with the following prototype of KKM type theorems:

Theorem A. Let $(E, D ; \Gamma)$ be an abstract convex space, $Y$ a topological space, and $F \in \mathfrak{K O}(E, Y)[r e s p$., $F \in \mathfrak{K C}(E, Y)]$. Let $G: D \multimap Y$ be a map such that

(A.1) for any $N \in\langle D\rangle, F\left(\Gamma_{N}\right) \subset G(N)$; and

(A.2) $G$ is open-valued [resp., closed-valued].

Then for each $N \in\langle D\rangle, F(E) \cap \bigcap\{G(y) \mid y \in N\} \neq \emptyset$. 


\section{Remark.}

1. If $E=Y$ and if the identity map $1_{E}=F \in \mathfrak{K}(E, E)$, then Condition (A.1) says that $G$ is a KKM map; see Theorem 0 .

2. If $E=Y=\Delta_{n}$ is an $n$-simplex, $D$ is the set of its vertices, and $\Gamma=$ co is the convex hull operation, then the celebrated KKM theorem [15] says that $1_{E} \in \mathfrak{K C}(E, E)$.

3. If $D$ is a nonempty subset of a topological vector space $E=Y$ (not necessarily Hausdorff), Fan's KKM lemma [7] says that $1_{E} \in \mathfrak{K C}(E, E)$.

4. For another forms of the KKM theorem for convex spaces, $H$-spaces, or $G$-convex spaces and their applications, there is a large number of works; see [8, 9, 14, 16, 21, 29] and references therein.

From Theorem A, we have another finite intersection property as follows:

Theorem B. Let $(E, D ; \Gamma)$ be an abstract convex space, $Y$ a topological space, and $F \in \mathfrak{K O}(E, Y)[$ resp., $F \in \mathfrak{K C}(E, Y)]$. Let $G: D \multimap Y$ and $H: E \multimap Y$ be maps satisfying

(B.1) $G$ is open-valued [resp., closed-valued];

(B.2) for each $x \in E, F(x) \subset H(x)$; and

(B.3) for each $y \in F(E), M \in\left\langle D \backslash G^{-}(z)\right\rangle$ implies $\Gamma_{M} \subset E \backslash H^{-}(z)$.

Then $F(E) \cap \bigcap\{G(z) \mid z \in N\} \neq \emptyset$ for all $N \in\langle D\rangle$.

The following coincidence theorem follows from Theorem B.

Theorem C. Let $(E, D ; \Gamma)$ be an abstract convex space, $Y$ a topological space, $S: D \multimap$ $Y, T: E \multimap Y$ maps, and $F \in \mathfrak{K O}(E, Y)[$ resp., $F \in \mathfrak{K} \mathfrak{C}(E, Y)]$. Suppose that

(C.1) $S$ is open-valued [resp., closed-valued];

(C.2) for each $y \in F(E), \operatorname{co}_{\Gamma} S^{-}(y) \subset T^{-}(y)$; and

(C.3) $F(E) \subset S(N)$ for some $N \in\langle D\rangle$.

Then there exists an $\bar{x} \in E$ such that $F(\bar{x}) \cap T(\bar{x}) \neq \emptyset$.

From Theorem C, we obtain the following Ky Fan type matching theorem:

Theorem D. Let $(E, D ; \Gamma)$ be an abstract convex space, $Y$ a topological space, $S: D \multimap$ $Y$, and $F \in \mathfrak{K O}(E, Y)[$ resp., $F \in \mathfrak{K C}(E, Y)]$ satisfying (C.1) and (C.3). Then there exists an $M \in\langle D\rangle$ such that $F\left(\Gamma_{M}\right) \cap \bigcap\{S(x) \mid x \in M\} \neq \emptyset$.

Theorem D can be stated in its contrapositive form and in terms of the complement $G(z)$ of $S(z)$ in $Y$. Then we obtain Theorem A. Therefore, Theorems A-D are equivalent. 


\section{KKM maps in metric spaces}

Let $(M, d)$ be a metric space. Motivated by Khamsi [11] and others, for a bounded subset $A \subset M$, we set

$\operatorname{ad}(A):=\bigcap\{B \mid B$ is a closed ball such that $A \subset B\}$ and

$\mathcal{A}(M):=\{A \subset M \mid A=\operatorname{ad}(A)\}$, i.e., $A \in \mathcal{A}(M)$ iff $A$ is an intersection of closed balls. In this case we will say $A$ is an admissible subset of $M$.

For a subset $A \subset M$ and $t>0$, let

$$
B_{M}(A, t):=\{x \in M \mid d(x, A) \leq t\} \text { and } N_{M}(A, t):=\{x \in M \mid d(x, A)<t\} .
$$

We introduce new definitions:

Definition. An abstract convex space $(M, D ; \Gamma)$ is called simply a metric space if $(M, d)$ is a metric space, $D$ is a nonempty set, and $\Gamma:\langle D\rangle \rightarrow \mathcal{A}(M)$ is a map having admissible values.

A $\Gamma$-convex subset of $(M \supset D ; \Gamma)$ is said to be subadmissible by some authors.

Example. We give examples of metric spaces $(M, D ; \Gamma)$ and KKM maps on them.

1. $(M \supset X ; \Gamma)$ where $\Gamma_{A}:=\operatorname{ad}(A)$; see [11]. A map $G: X \multimap M$ is called a KKM map if $\Gamma_{A} \subset G(A)$ for each $A \in\langle X\rangle$.

2. For each $A:=\left\{a_{0}, a_{1}, \ldots, a_{n}\right\} \in\langle D\rangle$, choose a subset $B:=\left\{x_{0}, x_{1}, \ldots, x_{n}\right\} \in\langle M\rangle$ and define $\Gamma_{A}:=\operatorname{ad}(B)$. Then $(M, D ; \Gamma)$ becomes a metric space. For this metric space, the so-called generalized $g K K M$ mapping in [6] simply becomes a KKM map.

Let $X$ be a nonempty subset of a metric space $(M, d)$. A map $F: X \multimap M$ is said to have the almost fixed point property (simply, a.f.p.p.) if for any $\varepsilon>0$, there exists an $x_{\varepsilon} \in X$ such that $F\left(x_{\varepsilon}\right) \cap B\left(x_{\varepsilon}, \varepsilon\right) \neq \emptyset$.

From Theorem $\mathrm{C}$, we deduce the following almost fixed point property of the $\mathfrak{K C}$-maps or $\mathfrak{K O}$-maps:

Theorem 4.1. Let $(M \supset D ; \Gamma)$ be a metric space and $X$ a $\Gamma$-convex subset of $M$ such that $X \cap D$ is dense in $X$. Suppose that $F \in \mathfrak{K C}(X, X)[$ resp., $\mathfrak{K O}(X, X)]$ such that $F(X)$ is totally bounded. Then $F$ has the a.f.p.p.

Proof 1. Let $E=Y:=X$ in Theorem C. Since $X \cap D$ is dense in $X$ and $F(X)$ is a totally bounded subset of $X$, for each $\varepsilon>0$, there exists a finite subset $A$ of $X \cap D$ such that $F(X) \subset \bigcup_{x \in A} N(x, \varepsilon) \subset \bigcup_{x \in A} B(x, \varepsilon)$. Let us define a map $S: X \cap D \multimap X$ by $S(x):=N(x, \varepsilon)$ [resp., $S(x):=B(x, \varepsilon)$ ] for $x \in X \cap D$. Then each $S(x)$ is open [resp., closed]. Therefore (C.1) and (C.3) are satisfied. Let us define another map $T: X \multimap X$ by $T(x):=B(x, \varepsilon)$ for $x \in X$. Then it is easy to show (C.2). [In fact, for any $N \in\left\langle S^{-}(y)\right\rangle$, 
$\Gamma_{N}$ is the intersection of closed balls containing $N$. Since $T^{-}(y)$ is one of such closed balls, we have $\Gamma_{N} \subset T^{-}(y)$.] Therefore, by Theorem C, there exists an $x_{\varepsilon} \in X$ such that $F\left(x_{\varepsilon}\right) \cap T\left(x_{\varepsilon}\right)=F\left(x_{\varepsilon}\right) \cap B\left(x_{\varepsilon}, \varepsilon\right) \neq \emptyset$.

Proof 2. Let $Y:=F(X)$. Then, for each $\varepsilon>0$, there exists a finite subset $A$ of $X \cap D$ such that $Y \subset \bigcup_{x \in A} N(x, \varepsilon) \subset \bigcup_{x \in A} B(x, \varepsilon)$. Let us define a map $G: X \multimap Y$ by $G(x):=$ $Y \backslash N(x, \varepsilon)$ [resp., $G(x):=Y \backslash B(x, \varepsilon)$ ] for $x \in X$. Then each $G(x)$ is closed [resp., open] and $\bigcap_{x \in A} G(x)=\emptyset$. Hence $G$ is not a KKM map with respect to $F \in \mathfrak{K C}(X, Y)$ [resp., $\mathfrak{K O}(X, Y)]$. Hence there exists a set $B=\left\{x_{0}, x_{1}, \ldots, x_{m}\right\} \in\langle X \cap D\rangle$ such that $F\left(\Gamma_{B}\right) \nsubseteq$ $G(B)$. Therefore, there exists an $x_{\varepsilon} \in F\left(\Gamma_{B}\right)$ such that $x_{\varepsilon} \notin G(B)$, that is, $x_{\varepsilon} \in N\left(x_{i}, \varepsilon\right)$ [resp., $\left.x_{\varepsilon} \in B\left(x_{i}, \varepsilon\right)\right]$ for all $i \in\{0,1, \ldots, m\}$. Hence $x_{i} \in N\left(x_{\varepsilon}, \varepsilon\right)$ [resp., $x_{\varepsilon} \in B\left(x_{i}, \varepsilon\right)$ ] for all $i \in\{0,1, \ldots, m\}$. If $x_{\varepsilon} \in F\left(x_{\varepsilon}^{\prime}\right)$ for some $x_{\varepsilon}^{\prime} \in \Gamma_{B} \subset B\left(x_{\varepsilon}, \varepsilon\right)$ or equivalently $x_{\varepsilon} \in \Gamma_{B} \subset B\left(x_{\varepsilon}^{\prime}, \varepsilon\right)$. Therefore, $x_{\varepsilon} \in F\left(x_{\varepsilon}^{\prime}\right) \cap B\left(x_{\varepsilon}^{\prime}, \varepsilon\right) \neq \emptyset$.

From Theorem 4.1, it is routine to deduce the following fixed point theorem:

Theorem 4.2. Let $(M \supset D ; \Gamma)$ be a metric space and $X$ a $\Gamma$-convex subset of $M$ such that $X \cap D$ is dense in $X$. Then any compact closed map $F \in \mathfrak{K C}(X, X)$ [resp., $\mathfrak{K O}(X, X)]$ has a fixed point.

In [29], we had the following:

Lemma 4.3. Let $(E, D ; \Gamma)$ be an abstract convex space, $Z, W$ two topological spaces, $F \in \mathfrak{K C}(E, Z)$ and $f: Z \rightarrow W$ a continuous function. Then $f F \in \mathfrak{K C}(E, W)$. This also holds for $\mathfrak{K O}$ instead of $\mathfrak{K C}$.

As a consequence of Theorem 4.2 and Lemma 4.3, we obtain a Schauder type fixed point theorem for metric spaces:

Corollary 4.4. Let $(M \supset D ; \Gamma)$ be a metric space and $X$ a $\Gamma$-convex subset of $M$ such that $X \cap D$ is dense in $X$. If the identity map $1_{E} \in \mathfrak{K C}(X, X)\left[\right.$ resp., $\left.1_{E} \in \mathfrak{K O}(X, X)\right]$, then any compact continuous function $f: X \rightarrow X$ has a fixed point.

Note that Theorems 4.1, 4.2, Lemma 4.3 and Corollary 4.4 properly generalize the corresponding ones in [1]. Moreover, the authors of [1] claimed that acyclic maps defined on $G$-convex spaces have the KKM property; but it was already shown by Park and Kim in 1997.

From Theorem 4.2 and Lemma 4.3, we have the following coincidence theorem:

Theorem 4.5. Let $(M \supset D ; \Gamma)$ be a metric space, $X$ a $\Gamma$-convex subset of $M$ such that $X \cap D$ is dense in $X$, and $Z$ a compact topological space. Let $T: Z \multimap X$ be a map having a continuous selection and $F \in \mathfrak{K C}(X, Z)$ [resp., $F \in \mathfrak{K O}(X, Z)]$ a closed map. Then there exist $x_{0} \in X$ and $z_{0} \in Z$ such that $z_{0} \in F\left(x_{0}\right)$ and $x_{0} \in T\left(x_{0}\right)$. 
Proof. Let $f$ be a continuous selection of $T$. Then $f F \in \mathfrak{K C}(X, X)$ by Lemma 4.3 and it is compact and u.s.c. Hence, by Theorem 4.2, it has a fixed point $x_{0} \in X$ such that $x_{0} \in f F\left(x_{0}\right)$. Therefore, $x_{0}=f\left(z_{0}\right) \in T\left(z_{0}\right)$ for some $z_{0} \in F\left(x_{0}\right)$.

From Theorem D, we deduce the following Ky Fan type matching theorem:

Theorem 4.6. Let $(M \supset D ; \Gamma)$ be a metric space, $X$ a $\Gamma$-convex subset of $M$, and $Z$ a topological space. Let $S: X \cap D \multimap Z$ be an open-valued [resp., closed-valued] map and $F \in \mathfrak{K C}(X, Z)[$ resp., $F \in \mathfrak{K O}(X, Z)]$ such that $F(X) \subset S(N)$ for some $N \in\langle X \cap D\rangle$. Then there exists an $A \in\langle X \cap D\rangle$ such that $F\left(\Gamma_{A}\right) \cap \bigcap\{T(x) \mid x \in A\} \neq \emptyset$.

Note that Theorems 4.5 and 4.6 are far-reaching extensions of [1, Theorem 2.5 and 2.7], resp.

From Theorem $\mathrm{C}$ with $E=Y$ and $F=1_{E}$, we have the following Fan-Browder type fixed point theorem:

Theorem 4.7. Let $(E, D ; \Gamma)$ be an abstract convex space such that $1_{E} \in \mathfrak{K O}(E, E)[$ resp., $\left.1_{E} \in \mathfrak{K C}(E, E)\right]$, and $S: D \multimap E, T: E \multimap E$ maps. Suppose that

(1) $S$ is open-valued [resp., closed-valued];

(2) for each $y \in E, \operatorname{co}_{\Gamma} S^{-}(y) \subset T^{-}(y)$; and

(3) $E=S(N)$ for some $N \in\langle D\rangle$.

Then there exists an $\bar{x} \in E$ such that $\bar{x} \in T(\bar{x})$.

Theorem 4.7 generalizes [1, Corollary 2.8].

In the last part of [1], the authors introduced $\mathcal{N} \mathcal{R}$-metric spaces, which are $G$-convex spaces and hence $K K M$-spaces. For those spaces, some known results on hyperconvex metric spaces are extended in [1].

\section{KKM maps in hyperconvex metric spaces}

Definition. A metric space $(H, d)$ is said to be hyperconvex if

$$
\bigcap_{\alpha} B\left(x_{\alpha}, r_{\alpha}\right) \neq \emptyset
$$

for any collection $\left\{B\left(x_{\alpha}, r_{\alpha}\right)\right\}$ of closed balls in $H$ for which $d\left(x_{\alpha}, x_{\beta}\right) \leq r_{\alpha}+r_{\beta}$.

Example. It is known that the space $\mathbb{C}(E)$ of all continuous real functions on a Stonian space $E$ (that is, extremally disconnected compact Hausdorff space) with the usual norm is hyperconvex, and that every hyperconvex real Banach space is a space $\mathbb{C}(E)$ for some Stonian space $E$. Therefore, $\left(\mathbf{R}^{n},\|\cdot\|_{\infty}\right), l^{\infty}$, and $L^{\infty}$ are concrete examples of hyperconvex metric spaces.

Results of Aronszajn and Panitchpakti [2, Theorem 1'] and Isbell [10, Theorem 1.1] are combined in the following: 
Lemma 5.1. A hyperconvex metric space is complete and (freely) contractible.

The following is easy to prove:

Lemma 5.2. An admissible subset of a hyperconvex metric space is hyperconvex.

Definition. An abstract convex space $(H, D ; \Gamma)$ is called simply a hyperconvex metric space if $(H, d)$ is a hyperconvex metric space, $D$ is a nonempty set, and $\Gamma:\langle D\rangle \rightarrow \mathcal{A}(H)$ is a map having admissible values such that

$$
A, B \in\langle D\rangle, A \subset B \text { implies } \Gamma_{A} \subset \Gamma_{B}
$$

There should be no confusion between a hyperconvex metric space $H=(H, d)$ and $(H, D ; \Gamma)$.

Theorem 5.3. Any hyperconvex metric space $(H, D ; \Gamma)$ is a KKM space.

Proof. For each $A \in\langle D\rangle, \Gamma_{A}$ is hyperconvex by Lemma 5.2 and hence contractible by Lemma 5.1. Therefore, $(H, D ; \Gamma)$ is an $H$-space and hence a $G$-convex space. It is well-known that any $G$-convex space is a KKM space.

\section{Example.}

1. As a consequence of Theorem 5.3, we obtain Khamsi's KKM theorem for a particular $\Gamma$ and for particular KKM maps with finitely closed values; see [11]. In fact, by replacing the original topology of $H$ by its finitely generated extension, we can eliminate "finitely".

2. From Theorem 5.3, we can obtain another particular forms in [3, Theorems 2 and 3].

It is well-known that any family of closed balls in a hyperconvex metric space has nonempty intersection whenever each two members of the family intersects. More precisely, we have the following [13, p.406]:

Lemma 5.4. (Penot) Let $(H, d)$ be a hyperconvex metric space and $\left\{A_{\alpha}\right\}_{\alpha \in \Lambda} \subset \mathcal{A}(H)$. If for each $\alpha, \beta \in \Lambda, A_{\alpha} \cap A_{\beta} \neq \emptyset$, then $\bigcap_{\alpha \in \Lambda} A_{\alpha} \neq \emptyset$.

Note that Lemma 5.4 simply tells that, for a family of admissible subsets of a hyperconvex metric space, the finite intersection property implies the whole intersection property. As was shown by the celebrated Ky Fan lemma [7], this fact is usually shown under some extra compactness assumption.

Theorem 5.5. Let $(E, D ; \Gamma)$ be an abstract convex space and $H$ a hyperconvex metric space. If $F \in \mathfrak{K C}(E, H)$ and $G: D \rightarrow \mathcal{A}(H)$ is a KKM map with respect to $F$, then $\bigcap_{z \in D} G(z) \neq \emptyset$. 
Proof. Since $F \in \mathfrak{K C}(E, H)$ and $G: D \rightarrow \mathcal{A}(H)$ is a closed-valued KKM map with respect to $F$, the family $\{G(z) \mid z \in D\} \subset \mathcal{A}(H)$ has the finite intersection property. Now, by Lemma 5.4, we have the conclusion.

Note that Theorem 5.5 reduces to [4, Theorem 5] and [5, Theorem 1] when $E=D$ is a metric space.

Corollary 5.6. [5, Corollary 1] Let $X$ be a $\Gamma$-convex subset of a hyperconvex metric space $(H \supset X ; \Gamma)$. If $F \in \mathfrak{K C}(X, X)$ and $G: X \cap D \rightarrow \mathcal{A}(X)$ is a KKM map with respect to $F$, then $G$ has a fixed point.

From Theorem B, we have another whole intersection property as follows:

Theorem 5.7. Let $(E, D ; \Gamma)$ be an abstract convex space and $H$ a hyperconvex metric space. Suppose that $F \in \mathfrak{K C}(E, H), S: D \rightarrow \mathcal{A}(H)$ and $T: E \multimap H$ are maps satisfying

(1) $S: D \rightarrow \mathcal{A}(H)$ is a KKM map with respect to $F$;

(2) for each $x \in E, F(x) \subset T(x)$; and

(3) for each $y \in F(E), M \in\left\langle D \backslash G^{-}(z)\right\rangle$ implies $\Gamma_{M} \subset E \backslash H^{-}(z)$.

Then $\bigcap_{z \in D} S(z) \neq \emptyset$.

Proof. Since $F \in \mathfrak{K C}(E, H)$ and $S: D \rightarrow \mathcal{A}(H)$ is a closed-valued KKM map with respect to $F$, by Theorem B, the family $\{S(z) \mid z \in D\} \subset \mathcal{A}(H)$ has the finite intersection property. Now, by Corollary 5.6, the family has the whole intersection property.

Theorem 5.8. Let $(E, D ; \Gamma)$ be an abstract convex space and $H$ a hyperconvex metric space. Suppose that

(1) $F \in \mathfrak{K C}(E, H)$;

(2) $G: D \multimap H$ is a KKM map with respect to $F$; and

(3) for each $z \in D, F(E) \cap G(z)$ is admissible in $H$.

Then $F(E) \cap \bigcap\{G(z) \mid z \in D\} \neq \emptyset$.

Proof. Define a map $G^{\prime}: D \rightarrow \mathcal{A}(H)$ by $G^{\prime}(z):=F(E) \cap G(z)$ for each $z \in D$. Since $F\left(\Gamma_{A}\right) \subset G(A)$ for each $A \in\langle D\rangle$ by (ii), we have

$$
F\left(\Gamma_{A}\right) \subset F(A) \cap G(A)=G^{\prime}(A)
$$

and hence, $G^{\prime}$ is a KKM map with respect to $F$. Then, by Theorem $5.5, \bigcap_{z \in D} G^{\prime}(z) \neq \emptyset$., which is the conclusion.

Theorem 5.8 reduces to $[5$, Theorem 2] whenever $E=D=X$ is a metric space and $F(X)$ and each $G(z)$ are admissible.

Remark. In Theorem 5.7, instead of (iii), we may assume

(iii) ${ }^{\prime} G$ has closed values and $F(X)$ is compact.

Applying Lemma 5.4, we obtain the following Ky Fan type matching theorem: 
Theorem 5.9. Let $(E, D ; \Gamma)$ be an abstract convex space, $H$ a hyperconvex metric space, $S: D \multimap H$, and $F \in \mathfrak{K C}(E, H)$ satisfying

(1) $S$ is open-valued;

(2) for each $z \in D, S^{c}(z):=H \backslash S(z)$ is admissible; and

(3) $H=S(D)$.

Then there exists an $A \in\langle D\rangle$ such that $F\left(\Gamma_{A}\right) \cap \bigcap\{S(z) \mid z \in A\} \neq \emptyset$.

Proof. Suppose, on the contrary, that $F\left(\Gamma_{A}\right) \subset \bigcup\left\{S^{c}(z) \mid z \in A\right\}$ for each $A \in\langle D\rangle$. Hence $S^{c}$ is a closed-valued KKM map with respect to $F$. Since $F \in \mathfrak{K C}(E, H)$, the family $\left\{S^{c}(z) \mid z \in D\right\}$ of admissible subsets of $H$ has the finite intersection property. Then by Lemma 5.4, we have $\bigcup\left\{S^{c}(z) \mid z \in D\right\} \neq \emptyset$. This contradicts (3).

Theorem 5.9 reduces to $[5$, Theorem 3] whenever $E=X$ is a metric space, $D=I$ is a subset of $X$, and $S(z)=U_{z}$.

The following coincidence theorem follows from Theorem 5.9:

Theorem 5.10. Let $(E, D ; \Gamma)$ be an abstract convex space, $H$ a hyperconvex metric space, $F \in \mathfrak{K C}(E, H)$ and $S: D \multimap H$ a map satisfying (1)-(3) of Theorem 5.8. Moreover, if

(4) $T: E \multimap H$ is a map satisfying $\operatorname{co}_{\Gamma} S^{-}(y) \subset T^{-}(y)$ for each $y \in H$, then there exists an $\bar{x} \in E$ such that $F(\bar{x}) \cap T(\bar{x}) \neq \emptyset$.

Proof. Applying Theorem 5.9, there exist an $A \in\langle D\rangle$, an $x_{0} \in \Gamma A$ and a $y_{0} \in F\left(x_{0}\right)$ such that $y_{0} \in \bigcap\{S(z) \mid z \in A\}$. Since $z \in A$ implies $z \in S^{-}\left(y_{0}\right)$, and hence $x_{0} \in \Gamma_{A} \subset$ $\operatorname{co}_{\Gamma} S^{-}\left(y_{0}\right) \subset T^{-}\left(y_{0}\right)$. Therefore $y_{0} \in F\left(x_{0}\right) \cap T\left(x_{0}\right)$.

Theorem 5.10 reduces to [4, Theorem 7] and [5, Theorem 4] whenever $E=D=X$ is a metric space.

In the last section of [5], its authors applied Theorem 5.5 to some abstract forms of generalized variational inequality problems. All of the results in [5, Section 3] can be generalized by our method.

The following is a Ky Fan type minimax inequality:

Theorem 5.11. Let $(E, D ; \Gamma)$ be an abstract convex space, $H$ a hyperconvex metric space, $F \in \mathfrak{K C}(E, H)$. Suppose that $\varphi: D \times H \rightarrow \mathbb{R}$ and $\psi: E \times H \rightarrow \mathbb{R}$ are real functions satisfying

(1) $\psi(x, y)<0$ for each $(x, y) \in \operatorname{Gr}(F)$;

(2) for each $z \in D,\{y \in H \mid \varphi(z, y) \leq 0\}$ is admissible; and

(3) for each $y \in H, \operatorname{co}_{\Gamma}\{z \in D \mid \varphi(z, y)>0\} \subset\{x \in E \mid \psi(x, y)>0\}$.

Then there exists a $y_{0} \in H$ such that $\varphi\left(z, y_{0}\right) \leq 0$ for each $z \in D$. 
Proof. Define two maps $T: E \multimap H$ and $G: D \multimap H$ by $T(x):=\{y \in H \mid \psi(x, y) \leq 0\}$ for $x \in E$ and $G(z):=\{y \in H \mid \varphi(z, y) \leq 0\}$ for $z \in D$.

By (1), we have $\operatorname{Gr}(F) \subset \operatorname{Gr}(T)$. By (2), each $G(z)$ is admissible. Moreover, by (3), for each $A \in\langle D\rangle$ and for each $y \in T\left(\Gamma_{A}\right)$,

$$
\varphi(z, y)>0 \text { for all } z \in A \text { implies } \psi(x, y)>0 \text { for all } x \in \Gamma_{A},
$$

or equivalently,

$$
\psi(x, y) \leq 0 \text { for some } x \in \Gamma_{A} \text { implies } \varphi(z, y) \leq 0 \text { for some } z \in A .
$$

Therefore $y \in T\left(\Gamma_{A}\right) \subset G(A)$ and hence $F\left(\Gamma_{A}\right) \subset G(A)$ for each $A \in\langle D\rangle$. Since $G: D \rightarrow \mathcal{A}(H)$ is a closed-valued KKM map with respect to $F \in \mathfrak{K C}(E, H)$, by Theorem 5.5 , we have a $y_{0} \in \bigcap_{z \in D} G(z)$, that is, $\varphi\left(z, y_{0}\right) \leq 0$ for each $z \in D$.

Theorem 5.11 reduces to $[5$, Theorem 5$]$ whenever $E=D=X$ is a metric space.

Similarly all of other results in Section 3 of [5] can be stated for an abstract convex spaces $(E, D ; \Gamma)$ instead of a metric space $(X ; \Gamma)$. Moreover, note that $[4]$ contains similar contents of $[5]$.

\section{References}

[1] A. Amini, M. Fakhar, and J. Zafarani, KKM mappings in metric spaces, Nonlinear Anal. TMA 66 (2007), 14-21.

[2] N. Aronszajn and P. Panitchpakdi, Extensions of uniformly continuous transformations and hyperconvex metric spaces, Pacific J. Math. 6 (1956), 405-439.

[3] T.-H. Chang, C.-M. Chen, and J.-H. Chang, Generalized 2-KKM theorems and their applications in hyperconvex metric spaces, Nonlinear Anal. TMA 69 (2008), 1145-1149.

[4] T.-H. Chang, C.-M. Chang, and H.-C. Huang, Generalized KKM theorems on hyperconvex metric spaces with applications, Taiwan. J. Math. 12(9) (2008), 2363-2372.

[5] T.-H. Chang, C.-M. Chen, and C.Y. Peng, Generalized KKM theorems on hyperconvex metric spaces and some applications, Nonlinear Anal. TMA 69 (2008), 530-535.

[6] C.-M. Chen and T.-H. Chang, Some results for the family ${ }_{2}^{2}{ }_{g} K K M(X, Y)$ and the $\Phi-$ mapping in hyperconvex metric spaces, Nonlinear Anal. TMA 69 (2008), 2533-2540.

[7] Ky Fan, A generalization of Tychonoff's fixed point theorem, Math. Ann. 142 (1961), 305310.

[8] C.D. Horvath, Contractibility and generalized convexity, J. Math. Anal. Appl. 156 (1991), 341-357.

[9] C.D. Horvath, Extension and selection theorems in topological spaces with a generalized convexity structure, Ann. Fac. Sci. Toulouse 2 (1993), 253-269.

[10] J. R. Isbell, Six theorems about injective metric spaces, Comment. Math. Helvatici 39 (1964), 439-447.

[11] M. A. Khamsi, KKM and Ky Fan theorems in hyperconvex metric spaces, J. Math. Anal. Appl. 204 (1996), 298-306. 
[12] W.A. Kirk and B. Panyanak, Best approximations in $\mathbb{R}$-trees, Numer. Funct. Anal. Optimiz. 28(5-6) (2007), 681-690.

[13] W. A. Kirk and B. Sims (ed.), Handbook of Metric Fixed Point Theory, Kluwer Academic Publ., Boston, 2001.

[14] W. A. Kirk, B. Sims, and G. X.-Z. Yuan, The Knaster-Kuratowski and Mazurkiewicz theory in hyperconvex metrix spaces and some of its applications, Nonlinear Anal. TMA 39 (2000), 611-627.

[15] B. Knaster, K. Kuratowski, S. Mazurkiewicz, Ein Beweis des Fixpunktsatzes für nDimensionale Simplexe, Fund. Math. 14 (1929), 132-137.

[16] M. Lassonde, On the use of KKM multifunctions in fixed point theory and related topics, J. Math. Anal. Appl. 97 (1983), 151-201.

[17] S. Park, Ninety years of the Brouwer fixed point theorem, Vietnam J. Math. 27 (1999), 193-232.

[18] S. Park, Fixed point theorems in hyperconvex metric spaces, Nonlinear Anal. TMA 37 (1999), 467-472.

[19] S. Park, The Schauder type and other fixed point theorems in hyperconvex spaces, Nonlinear Analysis Forum 3 (1998), 1-12.

[20] S. Park and B. Sims, Remarks on fixed point theorems on hyperconvex spaces, Nonlinear Funct. Anal. Appl. 5 (2000), 51-64.

[21] S. Park, Elements of the KKM theory for generalized convex spaces, Korean J. Comput. \& Appl. Math. 7 (2000), 1-28.

[22] S. Park, Coincidence, almost fixed point, and minimax theorems on generalized convex spaces, J. Nonlinear Convex Anal. 4 (2003), 151-164.

[23] S. Park, On generalizations of the KKM principle on abstract convex spaces, Nonlinear Anal. Forum 11 (2006), 67-77.

[24] S. Park, Fixed point theorems on $\mathfrak{K C}$-maps in abstract convex spaces, Nonlinear Anal. Forum 11(2006), 117-127.

[25] S. Park, Remarks on $\mathfrak{K C}-$ maps and $\mathfrak{K O}$-maps in abstract convex spaces, Nonlinear Anal. Forum 12(2007), 29-40.

[26] S. Park, Examples of $\mathfrak{K C}$-maps and $\mathfrak{K O}$-maps on abstract convex spaces, Soochow J. Math. 33(2007), 477-486.

[27] S. Park, A unified fixed point theory in generalized convex spaces, Acta Math. Sinica, English Series 23(2007), 1509-1536.

[28] S. Park, Various subclasses of abstract convex spaces for the KKM theory, Proc. Nat. Inst. Math. Sci. 2(2007), 35-47.

[29] S. Park, Elements of the KKM theory on abstract convex spaces, J. Korean Math. Soc. 45(2008), 1-27.

[30] S. Park, New foundations of the KKM theory, J. Nonlinear Convex. Anal. 9(2008), 331-350.

[31] S. Park, Remarks on the partial KKM principle, Nonlinear Anal. Forum 14 (2009), 51-62.

[32] S. Park and W. Lee, A unified approach to generalized KKM maps in generalized convex spaces, J. Nonlinear Convex Anal. 2 (2001), 157-166.

[33] R.C. Sine, On linear contraction semigroups in sup norm spaces, Nonlinear Anal. TMA 3 (1979), 885-890.

[34] P. Soardi, Existence of fixed points of nonexpansive mappings in certain Banach lattices, Proc. Amer. Math. Soc. 73 (1979), 25-29. 
The National Academy of Sciences, Republic of Korea, Seoul 137-044; and Department of Mathematical Sciences, Seoul National University, Seoul 151-747, Korea.

E-mail: shpark@math.snu.ac.kr 